\title{
Visualization of Protein-Protein Interaction Networks Using Force-Directed Layout
}

\author{
Yanga Byun and Kyungsook Han ${ }^{\star \star}$ \\ School of Computer Science and Engineering \\ Inha University, Inchon 402-751, Korea \\ quaah@hanmail.net, khan@inha.ac.kr \\ http://wilab.inha.ac.kr/protein/
}

\begin{abstract}
Protein interactions, when visualized as an undirected graph, often yield a nonplanar, disconnected graph with nodes of wide range of degrees. Many graph-drawing programs are of limited use in visualizing protein interactions, either because they are too slow, or because they produce a cluttered drawing with many edge crossings or a static drawing that is not easy to modify to reflect changes in data. We have developed a new force-directed layout algorithm for drawing protein interactions in three-dimensional space. Our algorithm divides nodes into three groups based on their interacting properties: biconnected subgraph in the center, terminal nodes at the outermost region, and the rest in between them. Experimental results show that our algorithm efficiently generates a clear and aesthetically pleasing drawing of large-scale protein interaction networks and that it is much faster than other force-directed layouts.
\end{abstract}

\section{Introduction}

Recent improvements in high-throughput proteomics technology such as yeast two-hybrid $[4,11]$ have produced a rapidly expanding volume of protein interaction data of an unprecedented scale. The interaction data is available either in text files or in databases. However, due to the volume of data (e.g., thousands of interacting proteins), a graphical representation of protein-protein interactions has proven to be much easier to understand than a long list of interacting proteins, prompting visualization studies of protein-protein interaction networks.

A Java applet program [6] has been developed for drawing protein interactions based on a relaxation algorithm and tested on the yeast two-hybrid $(\mathrm{Y} 2 \mathrm{H})$ data [11]. This program requires all protein-protein interaction data to be provided as parameters of the applet program in html sources. There is no way to save a visualized graph except by capturing the window. An image captured from the window is a static image and is of a generally low quality. It cannot be refined or changed later to reflect an update in data. A user can move a node

\footnotetext{
* This work was supported by the Ministry of Information and Communication of Korea under grant number IMT2000-C3-4.

** To whom correspondence should be addressed. email: khan@inha.ac.kr
} 
but cannot select or save a connected component containing a specific protein for later use. Recent work on yeast proteome also utilized a relaxation algorithm to visualize a protein complex network [3].

Other visualization works on protein interactions do not have their own algorithms or programs developed for visualization, but use general-purpose drawing tools. PSIMAP [7], for example, displays interactions between protein families by comparing the $\mathrm{Y} 2 \mathrm{H}$ data with the DIP data [12]. It was drawn by Tom Sawyer software (http://www.tomsawyer.com/) and then refined by significant amount of manual work to remove the edge crossings. From the perspective of graph drawing, PSIMAP is a static image and leaves several things to be improved. A research group of University of Washington $[9,10]$ has visualized the $\mathrm{Y} 2 \mathrm{H}$ data using another general-purpose drawing tool called AGD (http://www.mpisb.mpg.de/AGD/). Although AGD is powerful, it is a general-purpose drawing tool and does not provide a function that we hold are necessary for studying protein-protein interactions.

Existing visualization studies in protein interactions suggest that the nature of protein interaction data require a new graph layout method for protein interaction networks. Protein interaction data can be characterized as follows:

1. The data yields a nonplanar graph with a large number of edge crossings that cannot be removed in a two-dimensional drawing.

2. Proteins have a very wide range of interacting proteins within the same set of data, so a graph visualizing the data contains nodes of very high degree as well as those of low degree.

3. When visualized as a graph, the data yields a disconnected graph with many connected components. The MIPS genetic interaction data (http://mips.gsf. $\mathrm{de} / \mathrm{proj} /$ yeast/tables/interaction/), for example, contains 113 connected components.

4. The data often contains protein interactions corresponding to self-loops.

Considering these characteristics of protein interaction data, we have developed a new layout algorithm that divides nodes into three groups based on their interaction properties and layouts each group in three-dimensional space. The rest of this paper describes our algorithm and experimental results.

\section{A Partitioned Approach to Graph Drawing}

A common problem with many force-directed algorithms is that they become very slow when dealing with large graphs. We propose a new force-directed algorithm which divides nodes into three groups based on their interaction characteristics. Our layout is an extension of the algorithm by Kamada \& Kawai [5] for drawing two-dimensional graphs. Their original algorithm has been modified not only for three-dimensional graph drawing but also for improvements in the efficiency and resulting drawings of the algorithm. 


\subsection{Finding Groups}

Protein-protein interaction data can be visualized as an undirected graph $G=$ $(V, E)$, where nodes $V$ represent proteins and edges $E$ represent protein-protein interactions. The degree of a node $v_{i}$ is the number of its edges denoted by $\operatorname{deg}\left(v_{i}\right)$. An edge $e=\left(v_{i}, v_{j}\right)$ with $v_{i}=v_{j}$ is a self-loop. A cutvertex in a graph $G$ is a node whose removal disconnects $G$. A path in a graph $G$ is a sequence $\left(v_{1}, v_{2}, \ldots, v_{n}\right)$ of distinct nodes of $G$, such that $\left(v_{i}, v_{i+1}\right) \in E$ for $1 \leq i \leq n-1$. A graph $G^{\prime}=\left(V^{\prime}, E^{\prime}\right)$, such that $V^{\prime} \subseteq V$ and $E^{\prime} \subseteq E \cap\left(V^{\prime} \times V^{\prime}\right)$, is a subgraph of graph $G=(V, E)$. We divide nodes $V$ into three exclusive and exhaustive groups: $V_{1}, V_{2}, V_{3}$. The three groups are defined as follows:

- Group $V_{1}$ is a set of terminal nodes, i.e., nodes with degree 1.

- Group $V_{2}$ is a set of nodes in V-V1, which are in the subgraphs separated by cutvertices of degree $\geq 3$, except the nodes in the largest subgraph separated by the cutvertices.

- Group $V_{3}$ consists of nodes which are members of neither $V_{1}$ nor $V_{2}$.

Example 1 Consider a graph $G=(V, E)$ displayed in Fig. 1 . The nodes in $G$ are separated into three groups. Six nodes belong to $V_{1}$ and these are separated into three sub-groups, $V_{1}=\left\{\left\{v_{1}\right\},\left\{v_{5}, v_{9}, v_{10}\right\},\left\{v_{31}, v_{32}\right\}\right\}$. Each of the three sub-groups shares a neighbor.

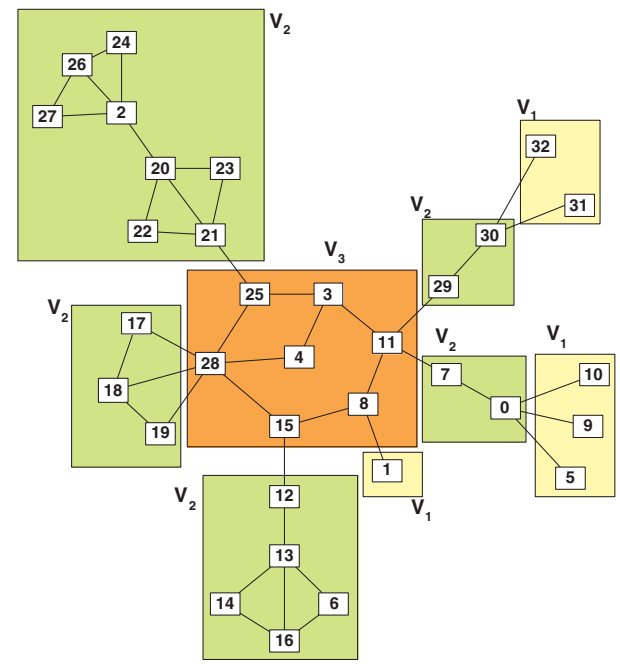

Fig. 1. Example of a partitioned graph. The nodes of $V_{1}$ are enclosed in yellow boxes, $V_{2}$ in green boxes, and $V_{3}$ in an orange box.

Example 2 In Fig. 1, two sub-groups $S_{1}=\left\{v_{0}, v_{7}\right\}$ and $S_{2}=\left\{v_{29}, v_{30}\right\}$ share a cutvertex $v_{11}$, so they are merged into one sub-group of $V_{2}$. Sub-groups $S_{3}=$ 
$\left\{v_{24}, v_{26}, v_{27}\right\}$ and $S_{4}=\left\{v_{2}, v_{20}, v_{21}, v_{22}, v_{23}, v_{24}, v_{26}, v_{27}\right\}$ do not share a cutvertex since the cutvertex of $S_{3}$ is $v_{2}$ and that of $S_{4}$ is $v_{25}$. However, $S_{3}$ is not counted as a sub-group of $V_{2}$ since $S_{3} \subset S_{4}$.
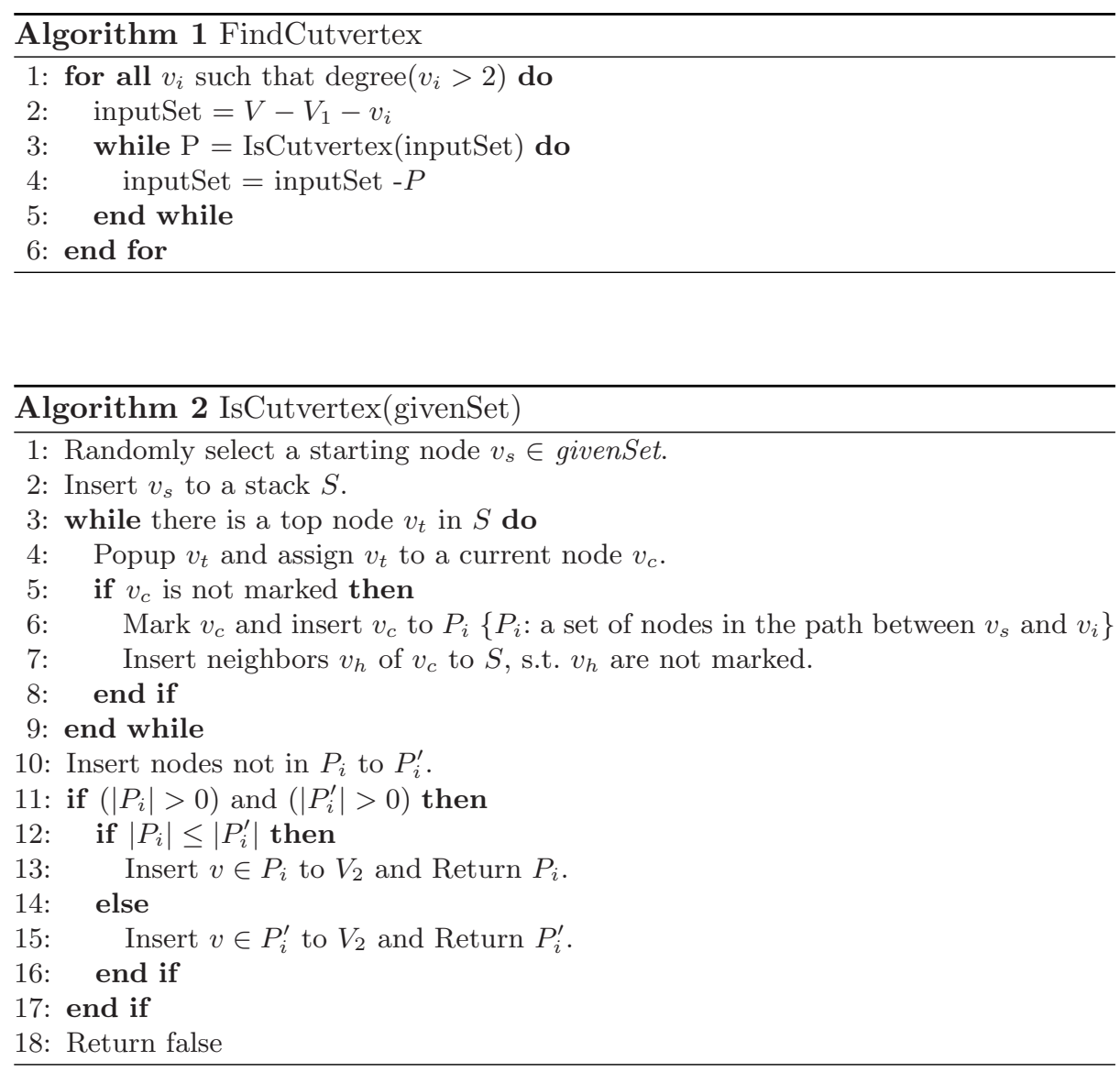

Nodes of each group are found in the order of $V_{1}, V_{2}$, and $V_{3}$. First, nodes with one neighbor are classified into $V_{1}$. Nodes of $V_{1}$ are further divided into sub-groups according to their shared neighbors. From $V-V_{1}$, nodes in $V_{2}$ are then found, and all remaining nodes constitute $V_{3}$.

After finding $V_{1}$, nodes of $V_{2}$ are determined by our heuristic algorithm FindCutvertex outlined in Algorithm 1. The initial input to the algorithm is nodes in $V-V_{1}$. For each input node $v_{i}$, the algorithm tests whether the node is a cutvertex (line 3 of Algorithm 1). Let $P$ be the set of nodes in the path between $v_{i}$ and the starting node and $P^{\prime}$ be the set of nodes not in the path. If neither of $P$ and $P^{\prime}$ is empty, the node $v_{i}$ is a cutvertex and the loop is repeated for the remaining nodes. The nodes in the smaller set between $P$ and $P^{\prime}$ are included 
in $V_{2}$ (lines 11-17 of Algorithm 2). The nodes of $V_{2}$ are further separated into sub-groups based on their cutvertex. These sub-groups are merged into one if they have the same cutvertex. All remaining nodes after determining both $V_{1}$ and $V_{2}$ constitute $V_{3} \cdot V_{3}$ corresponds to a biconnected subgraph (a connected graph with no cutvertices) in protein interaction data.

\subsection{Force-Directed Layout for Three-Dimensional Graph Drawing}

The algorithm by Kamada \& Kawai [5] searches for a drawing in which the energy is locally minimal. The aim of our algorithm is to find a drawing in which the actual distance between two nodes is approximately proportional to the desirable distance between them. The global energy of a spring system with $n$ nodes is defined by the equation:

$$
\begin{aligned}
E= & \sum_{i=1}^{n-1} \sum_{j=i+1}^{n} \frac{1}{2} k_{i j}\left(\left|p_{i}-p_{j}\right|-l_{i j}\right)^{2} \\
= & \sum_{i=1}^{n-1} \sum_{j=i+1}^{n} \frac{1}{2} k_{i j}\left\{\left(x_{i}-x_{j}\right)^{2}+\left(y_{i}-y_{j}\right)^{2}+\left(z_{i}-z_{j}\right)^{2}\right. \\
& \left.+l_{i j}^{2}-2 l_{i j} \sqrt{\left(x_{i}-x_{j}\right)^{2}+\left(y_{i}-y_{j}\right)^{2}+\left(z_{i}-z_{j}\right)^{2}}\right\}
\end{aligned}
$$

where $k_{i j}$ is the stiffness parameter of a spring, $p_{i}$ is the position of a node $v_{i}$, and $l_{i j}$ is the length at rest of the spring connecting $v_{i}$ and $v_{j}$.

The algorithm seeks a position $p_{m}=\left(x_{m}, y_{m}, z_{m}\right)$ for each vertex $v_{m}$ to minimize the potential energy in the spring system. Minima occur when the partial derivatives of $E$ with respect to each variables $x_{m}, y_{m}$, and $z_{m}$ are zero. This gives a set of $3|V|=3 n$ equations

$$
\frac{\partial E}{\partial x_{m}}=\frac{\partial E}{\partial y_{m}}=\frac{\partial E}{\partial z_{m}}=0, v_{m} \in V
$$

In Kamada \& Kawai's algorithm [5], a node is moved to a position that minimizes energy while all others remain fixed. The node to be moved is chosen as the one with the largest force acting on it, i.e., the one for which

$$
\sqrt{\left(\frac{\partial E}{\partial x_{m}}\right)^{2}+\left(\frac{\partial E}{\partial y_{m}}\right)^{2}+\left(\frac{\partial E}{\partial z_{m}}\right)^{2}}
$$

is maximized over all $v_{m} \in V$. However, this approach often yields unpleasant graphs and takes too much time for large-scale protein interactions. Thus, our algorithm moves all nodes to some level in each iteration until the difference between the current position and the previous position falls below a certain threshold value. For an initial layout we place nodes on the surface of a sphere instead of placing them randomly. Our algorithm yields more pleasant drawings than Kamada \& Kawai's algorithm and is much faster for graphs with balanced groups. 

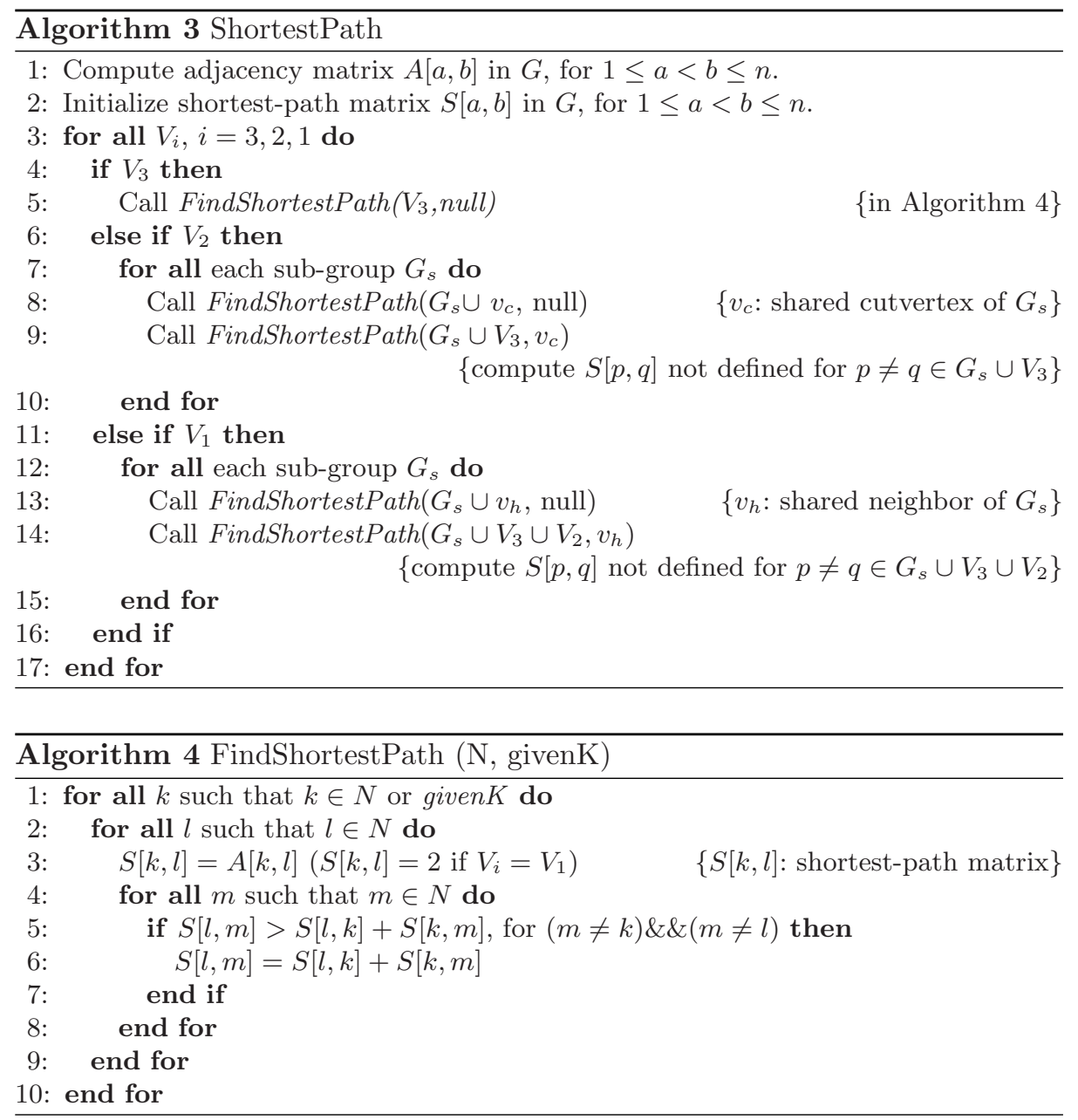

\subsection{Finding Shortest Paths in Groups}

The shortest path between every pair of nodes is computed for each group $V_{i}, i=1, \ldots, 3$. The algorithm for computing shortest paths is summarized in Algorithms 3 and 4 . We first compute shortest paths between nodes in $V_{3}$. For $V_{2}$ and $V_{1}$, shortest paths are determined in each of their sub-groups. After computing shortest paths between nodes in each sub-group, shortest paths between nodes of $V_{2}$ and nodes of $V_{3}$ are computed using a shared cutvertex of each subgroup of $V_{2}$ (line 9 of Algorithm 3). Likewise, shortest paths between nodes of $V_{1}$ and nodes of $V_{2}$ and $V_{3}$ are computed using a shared neighbor of each sub-group of $V_{1}$ (line 14). For a sub-group of $V_{1}$, the initial shortest path between every pair of nodes is set to 2 , since the distance between a node and its shared neighbor is 1 (line 3 of Algorithm 4). 


\section{Analytical and Empirical Evaluation}

Here we briefly analyze the computational cost of our algorithm. Assuming that three groups are balanced, the total time for our algorithm is $\left(\frac{n}{3}\right)^{3}+\left(\frac{n}{3}\right)^{3}+$ $\left(\frac{n}{3}\right)^{3}=\frac{n^{3}}{9}$ because we apply spring-embedder algorithm on each group. The asymptotic time complexity of our algorithm is the same as the time complexity $O\left(n^{3}\right)$ of Kamada \& Kawai's algorithm [5]. But our algorithm is much faster Kamada \& Kawai's algorithm in practice. Since the nodes in $V_{1}$ and $V_{2}$ are further divided into sub-groups, actual running times are reduced further for graphs with balanced groups. For a graph with unbalanced groups (for example, a graph in which the portion of $V_{3}$ is high due to few cutvertices or terminal nodes), the effect of dividing into three groups can be marginal, which is rare in protein interaction data. This has been supported by the experimental study to be discussed later.

Our algorithm was implemented in Microsoft C\#. The program runs on any PC with Windows 2000/XP/Me/98/NT 4.0 as its operating system. We tested the program on five cases, the PSIMAP data [7], Helicobacter pylori data [8], $\mathrm{Y} 2 \mathrm{H}$ data [11], and the data of protein interactions in yeast from the BIND (http://www.binddb.org/) and DIP (http://dip.doe-mbi.ucla.edu/) databases. In each of these data of protein interactions, the largest connected component was used for comparison. Table 1 shows the running times of our algorithm at each stage of partitioning nodes into three groups, finding shortest paths in all three groups, and layout and drawing.

Table 1. Running times of our algorithm at each stage. Hpylori: helicobacter pylori, $\mathrm{P}$ : partitioning nodes into $V_{1}, V_{2}$ and $V_{3}$, SP: finding shortest paths in all groups, LD: layout and drawing.

\begin{tabular}{|c|c|c|c|c|c|c|c|c|}
\hline \multirow{3}{*}{ Data } & \multirow{3}{*}{ Edges } & \multicolumn{3}{|c|}{ Nodes } & \multicolumn{4}{|c|}{ Running times } \\
\hline & & $V_{1}$ & $V_{2}$ & $V_{3}$ & $\mathrm{P}$ & SP & LD & total \\
\hline & & & & & (min: sec) & (min: sec) & (min: sec) & $(=P+S P+L D)$ \\
\hline$\overline{\mathrm{PSIMAP}}$ & 661 & 187 & 83 & 171 & 00:00.06 & $00: 00.04$ & 00:02.02 & $00: 02.12$ \\
\hline Hpylori & 1396 & 434 & 9 & 267 & 00:00.31 & 00:00.20 & 00:07.36 & 00:07.87 \\
\hline $\mathrm{Y} 2 \mathrm{H}$ & 12909 & 3066 & 28 & 642 & $00: 26.78$ & $00: 34.38$ & $09: 12.25$ & $10: 13.41$ \\
\hline BIND & 8243 & 3039 & 45 & 893 & $00: 15.67$ & $00: 26.02$ & $08: 21.82$ & 09:03.51 \\
\hline DIP & 14415 & 3278 & 126 & 1195 & 00:30.00 & $00: 43.45$ & $10: 41.64$ & $11: 55.09$ \\
\hline
\end{tabular}

Fig. 2A shows an initial layout by our algorithm for the $\mathrm{Y} 2 \mathrm{H}$ data with 12909 interactions between 3736 proteins. While we find groups in the order of $V_{1}, V_{2}$, and $V_{3}$, we layout them in reverse order; $V_{3}$ is first positioned in the center of the sphere, $V_{2}$ in the outer region of $V_{3}$, and $V_{1}$ in the outer region of $V_{2}$ and $V_{3}$. Groups for which node positions are fixed are shown in a rectangle. Nodes in remaining groups are relocated with modified polar coordinates in order to place them in the outer region of the groups that have been fixed. In Figs 2B 
(A)

(B)
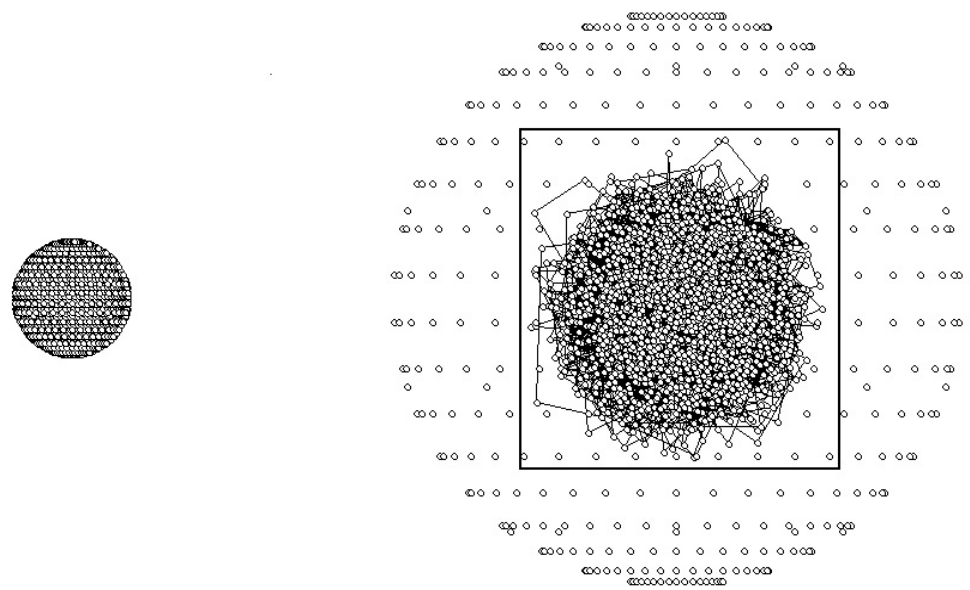

(C)

(D)
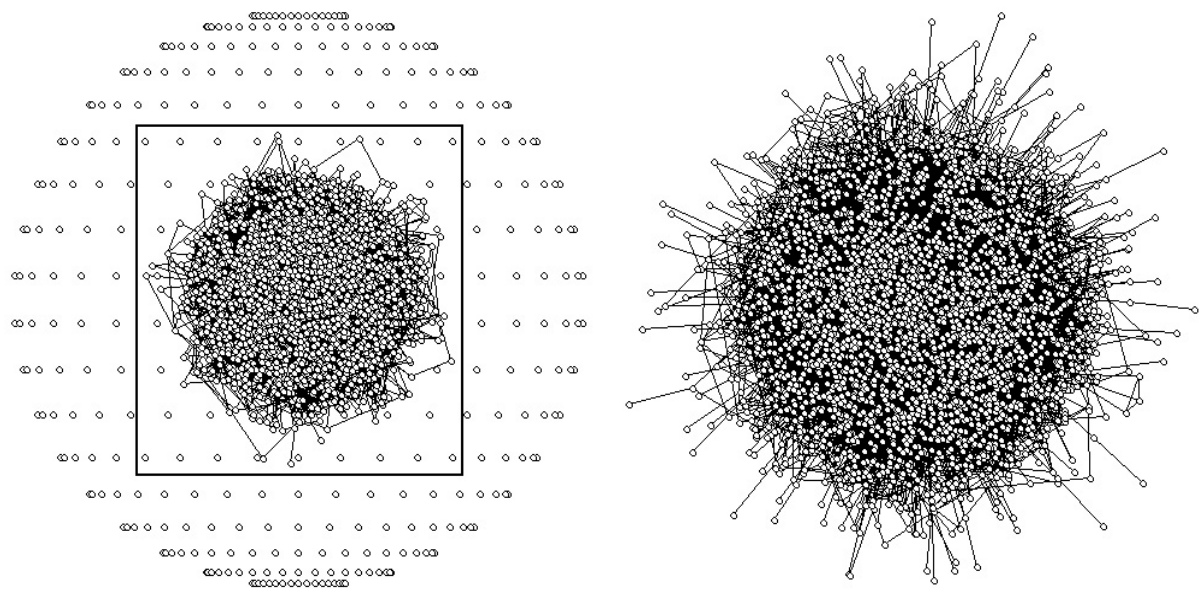

Fig. 2. Drawings of the Y2H data with 12909 interactions between 3736 proteins. (A) Initial layout, (B) After drawing the nodes in $V_{3}$ shown in the rectangle, (C) After drawing the nodes in $V_{3}$ and $V_{2}$ shown in the rectangle, (D) Final drawing. 
and $2 \mathrm{C}$, edges between nodes in the outer area are not shown for the clarity of the drawing.

For the purpose of experimental comparison with other algorithms, we also ran Pajek [1] with Fruchterman \& Reingold's algorithm [2] and our own implementation of Kamada \& Kawai's algorithm [5]. Since Kamada \& Kawai's algorithm produces a two-dimensional drawing only, we extended their algorithm to a three-dimensional drawing. Table 2 shows the running times of our algorithm, Kamada \& Kawai's algorithm extended to 3D, and Fruchterman \& Reingold's algorithm on five test cases on a Pentium IV 1.5Ghz processor. With our partitioning method, the computation time can be significantly reduced. The running times of the three algorithms are also plotted in Fig. 3. It follows from this result that our algorithm is more effective for bigger graphs and for graphs with balanced groups.

Table 2. Running times of graph drawing algorithms on 5 test cases on a Pentium IV 1.5Ghz processor. K-K extended to 3D: Kamada \& Kawai's algorithm extended for 3D drawing. Pajek (F-R): Pajek with Fruchterman \& Reingold's layout.

\begin{tabular}{|c|c|c|c|c|c|}
\hline Data & Nodes & Edges & Our algorithm & K-K extended to $3 \mathrm{D}$ & Pajek (F-R) \\
\hline$\overline{\text { PSIMAP }}$ & 441 & 661 & $00 \mathrm{~m} 02.12 \mathrm{~s}$ & $00 \mathrm{~m} \mathrm{07.69s}$ & $00 \mathrm{~m} \mathrm{33s}$ \\
\hline Hpylori & 710 & 1396 & $00 \mathrm{~m} 07.87 \mathrm{~s}$ & $00 \mathrm{~m} 22.14 \mathrm{~s}$ & $01 \mathrm{~m} \mathrm{14s}$ \\
\hline $\mathrm{Y} 2 \mathrm{H}$ & 3736 & 12909 & $10 \mathrm{~m} \mathrm{13.41s}$ & $13 \mathrm{~m} 23.47 \mathrm{~s}$ & $32 \mathrm{~m} \mathrm{10s}$ \\
\hline BIND & 3977 & 8243 & $09 \mathrm{~m} 03.51 \mathrm{~s}$ & $14 \mathrm{~m} 24.39 \mathrm{~s}$ & $41 \mathrm{~m} 37 \mathrm{~s}$ \\
\hline DIP & 4599 & 14415 & $11 \mathrm{~m} 55.09 \mathrm{~s}$ & $19 \mathrm{~m} \mathrm{09.81s}$ & $55 \mathrm{~m} 55 \mathrm{~s}$ \\
\hline
\end{tabular}

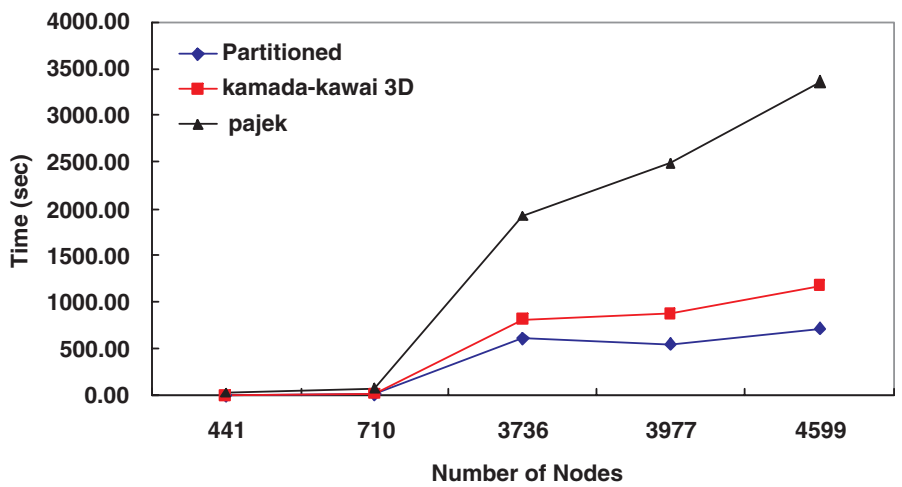

Fig. 3. Running times of three graph drawing algorithms. 


\section{Discussion and Conclusions}

Many force-directed graph drawing algorithms are too slow to be used for visualizing large-scale protein interactions and they often yield unclear drawings with many edge crossings. This paper presented a new algorithm for drawing large-scale protein interaction networks in three-dimensional space. The algorithm divides nodes into three groups: a biconnected subgraph in the center of a graph $\left(V_{3}\right)$, terminal nodes at the outermost region $\left(V_{1}\right)$, and the rest in between them $\left(V_{2}\right)$. These groups are identified from the outer group $\left(V_{1}\right)$ to inner one $\left(V_{3}\right)$ and are placed in reverse order. Experimental results demonstrate that the algorithm generates clear and aesthetically pleasing drawings of both large and small scale graphs and that for graphs with balanced groups it is significantly faster than other force-directed algorithms.

\section{References}

[1] V. Batagelj and A. Mrvar. Pajek - analysis and visualization of large networks. Lecture Notes in Computer Science, 2265:477-478, 2001.

[2] T.M.J. Fruchterman and E.M. Reingold. Graph drawing by force-directed placement. Software-Practice and Experience, 21(11):1129-1164, 1991.

[3] A.-C Gavin, M. Bosche, R. Krause, and et al. Functional organization of the yeast proteome by systematic analysis of protein complexes. Nature, 415(6868):141-147, 2002.

[4] T. Ito, K. Tashiro, S. Muta, R. Ozawa, T. Chiba, M. Nishizawa, K. Yamamoto, S. Kuhara, and Y. Sakaki. Toward a protein-protein interaction network of the budding yeast: A comprehensive system to examine two-hybrid interactions in all possible combinations between the yeast proteins. Proc. Natl. Acad. Sci. USA, 97(3):1143-1147, 2000.

[5] T. Kamada and S. Kawai. An Algorithm for Drawing General Undirected Graphs. Information Processing Letters, 31:7-15, 1989.

[6] R. Mrowka. A java applet for visualizing protein-protein interaction. Bioinformatics, 17(7):669-670, 2001.

[7] J. Park, M. Lappe, and S.A. Teichmann. Mapping protein family interactions: intramolecular and intermolecular protein family interaction repertoires in the PDB and Yeast. J. Mol. Biol., 307:929-938, 2001.

[8] J.-C. Rain, L. Selig, H.D. Reuse, V. Battaglia, C. Reverdy, S. Simon, G. Lenzen, F. Petel, J. Wojcik, V. Schachter, Y. Chemama, A. Labigne, and P. Legrain. The protein-protein interaction map of helicobacter pylori. Nature, 409:211-215, 2001.

[9] B. Schwikowski, P. Uetz, and S. Fields. A network of protein-protein interactions in yeast. Nature Biotechnology, 18(12):1257-1261, 2000.

[10] C.L. Tucker, J.F. Gera, and P. Uetz. Towards an understanding of complex protein networks. Trends in Cell Biology, 11(3):102-106, 2001.

[11] P. Uetz, L. Giot, G. Cagney, and et al. A comprehensive analysis of protein-protein interactions in Saccharomyces cerevisiae. Nature, 403:623-627, 2000.

[12] I. Xenarios, E. Fernandez, L. Salwinski, X.J. Duan, M.J. Thompson, E.M. Marcotte, and D. Eisenberg. DIP: the database of interacting proteins: 2001 update. Nucleic Acids Res., 29(1):239-241, 2001. 